\title{
Self-similar Random Vector Fields and Their Wavelet Analysis
}

\author{
Pouya Dehghani Tafti and Michael Unser
}

\begin{abstract}
This paper is concerned with the mathematical characterization and wavelet analysis of self-similar random vector fields. The study consists of two main parts: the construction of random vector models on the basis of their invariance under coordinate transformations, and a study of the consequences of conducting a wavelet analysis of such random models. In the latter part, after briefly examining the effects of standard wavelets on the proposed random fields, we go on to introduce a new family of Laplacian-like vector wavelets that in a way duplicate the covariant-structure and whitening relations governing our random models.
\end{abstract}

\section{INTRODUCTION}

Self-similar or fractal random processes and fields have been used for modelling a wide range of man-made and natural phenomena. ${ }^{1}$ This is to a large extent due to their special form of scale-invariant behaviour (self-similarity) that is either strictly or approximately obeyed by the phenomena in question. This form of scale-invariance is also central to the definition of wavelets. Wavelets therefore come across as natural tools for the analysis of data obtained from the observation of fractal phenomena and for quantifying their correspondence to the said models. ${ }^{2-7}$

The most prominent example of self-similar random fields is fractional Brownian motion (fBm). ${ }^{8}$ While classical fractional Brownian fields typically correspond to physical scalars, a vectorial extension of such models can be envisioned by bringing in certain laws of coordinate transformation that govern vector quantities in physics. In the present work we first introduce such a vector extension of fractional Brownian fields, and then review the analysis of these random models using existing, as well as novel, wavelets.

To obtain a physically relevant stochastic vector model, we shall focus on two important categories of coordinate transformations-namely rotations and scalings—and study $\mathbb{R}^{d}$-valued self-similar random fields that rotate according to the laws of coordinate transformation that hold true for vector fields. The desired properties are found in a pseudo-differential operator (an extension of the vector Laplacian), which is used to define the vector fBm field $\mathfrak{B}_{H, \boldsymbol{\xi}}$ via a whitening equation:

$$
(-\boldsymbol{\Delta})_{\boldsymbol{\xi}}^{\frac{H}{2}+\frac{d}{4}} \mathfrak{B}_{H, \boldsymbol{\xi}}=\epsilon_{H} \mathfrak{W}
$$

$\left(\mathfrak{W}\right.$ is a vector of independent and normalized white noise fields and $\epsilon_{H}$ is a constant).

The above equation is interpreted in Gel'fand and Vilenkin's framework for distribution-theoretical stochastic analysis. ${ }^{9}$ In this formalism, random fields are defined by specifying the joint probability measures of their 'inner-products' with test functions. This process can also be understood as taking the random field to be a generalized function (distribution) chosen at random from a generalized function space.

In the following sections we first briefly review the definition of the extended fractional Laplacian operators mentioned above, and then use them to define vector $\mathrm{fBm}$ fields. Wavelet analysis of vector $\mathrm{fBm}$ takes up the remainder of the paper, and is divided in two parts: an analysis using standard wavelets comes first; this is then followed by a novel vector wavelet construction that is also used to analyze the random fields in question.

\section{FRACTIONAL LAPLACIANS AND THEIR INVERSES}

The Laplacian operator $(-\boldsymbol{\Delta})_{\xi}^{\gamma}$ that appears in (1) is linked to the matrix-valued function

$$
\boldsymbol{\Phi}_{\left(\xi_{1}, \xi_{2}\right)}^{\gamma}(\boldsymbol{x}):=\|\boldsymbol{x}\|^{2 \gamma}\left[\mathrm{e}^{\xi_{1}} \frac{\boldsymbol{x} \boldsymbol{x}^{\top}}{\|\boldsymbol{x}\|^{2}}+\mathrm{e}^{\xi_{2}}\left(\mathbf{I}-\frac{\boldsymbol{x} \boldsymbol{x}^{\top}}{\|\boldsymbol{x}\|^{2}}\right)\right],
$$

Wavelets XIII, edited by Vivek K. Goyal, Manos Papadakis, Dimitri Van De Ville, Proc. of SPIE Vol. 7446, 74460Y · @ 2009 SPIE · CCC code: 0277-786X/09/\$18 · doi: 10.1117/12.824873 
via the equation

$$
(-\boldsymbol{\Delta})_{\xi}^{\gamma} \boldsymbol{f}:=(2 \pi)^{-d} \int_{\mathbb{R}^{d}} \mathrm{~d} \boldsymbol{\omega} \mathrm{e}^{\mathrm{j} \boldsymbol{x}^{\top} \boldsymbol{\omega}} \boldsymbol{\Phi}_{\boldsymbol{\xi}}^{\gamma}(\boldsymbol{\omega}) \hat{\boldsymbol{f}}(\boldsymbol{\omega})
$$

for $\gamma \in \mathbb{R}_{+}$and $\mathrm{e}^{\xi_{1}}, \mathrm{e}^{\xi_{2}} \in \mathbb{R}$.

To gain a better understanding of the action of the extended fractional Laplacian let us introduce the auxiliary operator

$$
\mathbf{E}: \boldsymbol{f} \mapsto(2 \pi)^{-d} \int_{\mathbb{R}^{d}} \mathrm{~d} \boldsymbol{\omega} \mathrm{e}^{\mathrm{j} \boldsymbol{x}^{\top} \boldsymbol{\omega}} \frac{\boldsymbol{\omega} \boldsymbol{\omega}^{\top}}{\|\boldsymbol{\omega}\|^{2}} \hat{\boldsymbol{f}}(\boldsymbol{\omega}) ;
$$

whereby

$$
(-\boldsymbol{\Delta})_{\boldsymbol{\xi}}^{\gamma}=\mathrm{e}^{\xi_{1}}(-\boldsymbol{\Delta})_{\mathbf{0}}^{\gamma} \mathbf{E}+\mathrm{e}^{\xi_{2}}(-\boldsymbol{\Delta})_{\mathbf{0}}^{\gamma}(\mathrm{Id}-\mathbf{E}) .
$$

$\mathbf{E}$ and its complement (Id - E) project their argument onto the curl-free, and divergence-free, subspaces of $\left(\mathrm{L}^{d}\right)^{d}$ respectively. This observation clarifies the action of the extended Laplacian $(-\boldsymbol{\Delta})_{\xi}^{\gamma}$, which can thus be said to consists of an ordinary fractional vector Laplacian-operating independently on each component of the field-combined with a Helmholtz-like decomposition and weighted recombination, with the weights depending directly on $\boldsymbol{\xi}=\left(\xi_{1}, \xi_{2}\right)$.

It can therefore be seen that the parameters $\xi_{1}$ and $\xi_{2}$ govern the vectorial behaviour of the solution to (1). As important special cases, in addition to the classical fractional Brownian motion obtained by choosing $\xi_{1}=\xi_{2}$, divergence-free (solenoidal) and curl-free (irrotational) solutions to (1) can be produced by letting $\xi_{1} \rightarrow \infty$ and $\xi_{2} \rightarrow \infty$ respectively.

Our interest in extended fractional Laplacians is rooted in the fact that they interact with coordinate transformations in a specific way: given the transformation operators

$$
\mathbf{S}_{\sigma}: \boldsymbol{f}(\bullet) \mapsto \boldsymbol{f}\left(\sigma^{-1} \bullet\right)
$$

and

$$
\mathrm{R}_{\Omega}: f(\bullet) \mapsto \Omega f\left(\Omega^{\top} \bullet\right),
$$

(vector-field rotation)

one can verify that the following 'quasi-invariances' hold.

$$
(-\boldsymbol{\Delta})_{\xi}^{\gamma} \mathbf{S}_{\sigma} \boldsymbol{f}=\sigma^{2 \gamma} \mathbf{S}_{\sigma}(-\boldsymbol{\Delta})_{\xi}^{\gamma} \boldsymbol{f}
$$

and

$$
(-\Delta)_{\xi}^{\gamma} \mathbf{R}_{\Omega} f=\mathbf{R}_{\Omega}(-\Delta)_{\xi}^{\gamma} f
$$

(rotation-invariance)

( $\Omega$ denotes an arbitrary rotation or reflection matrix.)

\subsection{Inverse Operators}

To solve (1) for a self-similar solution one needs to invert $(-\boldsymbol{\Delta})_{\boldsymbol{\xi}}^{\gamma}$ subject to zero boundary conditions at $\boldsymbol{x}=\mathbf{0}$. This process gives rise to the inverse operator

$$
(-\dot{\Delta})_{-\xi}^{-\gamma}: \boldsymbol{f} \mapsto \frac{1}{(2 \pi)^{d}}\left(\mathrm{e}^{\mathrm{j}\langle\boldsymbol{x}, \boldsymbol{\omega}\rangle}-\sum_{|\boldsymbol{k}| \leq\left\lfloor 2 \gamma-\frac{d}{2}\right\rfloor} \frac{\mathrm{j}^{|\boldsymbol{k}|} \boldsymbol{x}^{\boldsymbol{k}} \boldsymbol{\omega}^{\boldsymbol{k}}}{\boldsymbol{k} !}\right) \Phi_{-\boldsymbol{\xi}}^{-\gamma}(\boldsymbol{\omega}) \hat{\boldsymbol{f}}(\boldsymbol{\omega}) \mathrm{d} \boldsymbol{\omega} .
$$

As already mentioned in the introduction, in our distribution-theoretical formalism the self-similar solution to (1), that is, the random field

$$
\mathfrak{B}_{H, \boldsymbol{\xi}}:=\epsilon_{H}(-\dot{\boldsymbol{\Delta}})_{-\boldsymbol{\xi}}^{-\gamma} \mathfrak{W},
$$


is defined in terms of 'inner products' $\left\langle\epsilon_{H}(-\dot{\boldsymbol{\Delta}})_{-\xi}^{-\gamma} \mathfrak{W}, \boldsymbol{g}\right\rangle$ with test functions $\boldsymbol{g}$. We shall demonstrate in the next section that an exact meaning can be attached to these inner products by transposing the operator to the right side. For this purpose, it is necessary to identify the dual of the above inverse operator; i.e., the operator $(-\grave{\Delta})_{-\bar{\xi}}^{-\gamma}$ satisfying

$$
\left\langle(-\dot{\Delta})_{-\xi}^{-\gamma} \boldsymbol{f}, \boldsymbol{g}\right\rangle=\left\langle\boldsymbol{f},(-\grave{\boldsymbol{\Delta}})_{-\overline{\boldsymbol{\xi}}}^{-\gamma} \boldsymbol{g}\right\rangle
$$

for all test functions $f$ and $g$. This dual operator is given by the integral

$$
(-\grave{\boldsymbol{\Delta}})_{-\boldsymbol{\xi}}^{-\gamma}: \boldsymbol{f} \mapsto(2 \pi)^{-d} \int_{\mathbb{R}^{d}} \mathrm{~d} \boldsymbol{\omega} \mathrm{e}^{\mathrm{j} \boldsymbol{x}^{\top} \boldsymbol{\omega}} \boldsymbol{\Phi}_{-\boldsymbol{\xi}}^{-\gamma}(\boldsymbol{\omega})\left[\mathbf{R}^{\gamma} \hat{\boldsymbol{f}}\right](\boldsymbol{\omega})
$$

with the regularizer $\mathbf{R}^{\gamma}$ defined by

$$
\mathbf{R}^{\gamma}: \hat{\boldsymbol{f}}(\bullet) \mapsto \hat{\boldsymbol{f}}(\bullet)-\sum_{|\boldsymbol{k}| \leq\left\lfloor 2 \gamma-\frac{d}{2}\right\rfloor} \mathbf{T}_{\boldsymbol{k}}[\hat{\boldsymbol{f}}](\bullet)^{k},
$$

where $\mathbf{T}_{\boldsymbol{k}}[\hat{\boldsymbol{f}}]$ denotes the (vector) coefficient of $(\bullet)^{\boldsymbol{k}}$ in the Taylor series expansion of $\hat{\boldsymbol{f}}(\bullet)$ around $\mathbf{0 .}$

\section{VECTOR FRACTIONAL BROWNIAN MOTION}

The properties of statistical self-similarity and invariance to domain rotations are at the heart of the various definitions of vector fractional Brownian motion we shall now consider.

\subsection{Vector Fractional Brownian Motion}

Fractional Brownian motions (fBm's) are defined as specific types of non-stationary zero-mean Gaussian fields. As a result of their Gaussian probability law, knowledge of their second-order statistics suffices for their full characterization. A vector $\mathrm{fBm} \mathfrak{B}_{H}$ with Hurst exponent $H, 0<H<1$ is classically defined via its variogram, i.e., the expectation

$$
\mathbf{E}\left\{\left\|\mathfrak{B}_{H}(\boldsymbol{x})-\mathfrak{B}_{H}(\boldsymbol{y})\right\|^{2}\right\} \propto\|\boldsymbol{x}-\boldsymbol{y}\|^{2 H} .
$$

Self-similarity and rotation-invariance of $\mathfrak{B}_{H, \boldsymbol{\xi}}$ can be directly verified in the above formula.

The above definition leaves the cross-correlation of vector components unspecified. These components are normally assumed to be independent, in which case vector $\mathrm{fBm}$ becomes nothing but a vector of scalar $\mathrm{fBm}$ 's. ${ }^{10}$ When considering specific forms of vector behaviour (such as divergence-free flow), this independence assumption proves to be too restrictive and, consequently, a broader definition is to be sought.

Such a broad definition of vector $\mathrm{fBm}$ was given in the introduction by means of a whitening equation (Eqn (1)), that is, a stochastic fractional PDE of the form

$$
(-\boldsymbol{\Delta})_{\xi}^{\frac{H}{2}+\frac{d}{4}} \mathfrak{B}_{H, \boldsymbol{\xi}}=\epsilon_{H} \mathfrak{W} ;
$$

where $\epsilon_{H}$ is a constant, $\mathfrak{W}$ is a vector of normalized white noise fields, and $\mathfrak{B}_{H, \boldsymbol{\xi}}$ denotes the desired solution. The extended Laplacian operator $(-\boldsymbol{\Delta})_{\boldsymbol{\xi}}^{\gamma}$ defined in the previous section accounts for the self-similarity and rotation-invariance of $\mathfrak{B}_{H, \boldsymbol{\xi}}$. To give an explicit definition of $\mathfrak{B}_{H, \boldsymbol{\xi}}$ the operator needs to be inverted with attention to the special boundary conditions imposed on the solution (zero boundary conditions at $\boldsymbol{x}=\mathbf{0}$ ). This is achieved using the first of the particular inverse operators introduced in §2.1:

$$
\mathfrak{B}_{H, \boldsymbol{\xi}}:=\epsilon_{H}(-\dot{\boldsymbol{\Delta}})_{-\boldsymbol{\xi}}^{-\frac{2 H+d}{4}} \mathfrak{W}
$$

One has command over the order of self-similarity of the resulting random models via the parameter $H$ (the Hurst exponent). Directional behaviour (i.e., solenoidal vs irrotational tendencies) can also be controlled by adjusting $\xi$, as noted in $\S 2$. These models can therefore be adapted to the directional structure of the phenomenon under investigation. 


\subsection{Mathematical Interpretation}

We shall at this point elaborate briefly on the mathematical interpretation of Eqn (3). In Gel'fand and Vilenkin's theory of stochastic analysis, one takes a random function such as $\mathfrak{W}$ to be an entity whose 'inner products' $\langle\mathfrak{W}, \boldsymbol{g}\rangle$ with test functions $\boldsymbol{g}$ in some function space are ordinary random variables. $\mathfrak{W}$ is then fully defined once the joint probability measure of any finite number of such inner products is given in a consistent manner. If an operator such as $(-\boldsymbol{\Delta})_{-\boldsymbol{\xi}}^{-\frac{2 H+d}{4}}$ acts on $\mathfrak{W}$, this operator is carried over to the side of the test function by duality, yielding the equality

$$
\left\langle(-\dot{\Delta})_{-\boldsymbol{\xi}}^{-\frac{2 H+d}{4}} \mathfrak{W}, \boldsymbol{g}\right\rangle=\left\langle\mathfrak{W},(-\grave{\Delta})_{-\boldsymbol{\xi}}^{-\frac{2 H+d}{4}} \boldsymbol{g}\right\rangle
$$

that holds by definition.

As noted previously, when dealing with zero-mean Gaussian random functions (as in the present case), knowledge of second-order statistics suffices. For the vector $\mathrm{fBm} \mathfrak{B}_{H, \boldsymbol{\xi}}$ this information is captured by the correlation form defined by the equation

$$
\langle\langle\boldsymbol{f}, \boldsymbol{g}\rangle\rangle_{\mathfrak{B}_{H, \boldsymbol{\xi}}}:=\mathbf{E}\left\{\overline{\left\langle\boldsymbol{B}_{H, \boldsymbol{\xi}}, \boldsymbol{f}\right\rangle}\left\langle\boldsymbol{\mathfrak { B }}_{H, \boldsymbol{\xi}}, \boldsymbol{g}\right\rangle\right\} .
$$

This quantity can be computed as follows:

$$
\begin{aligned}
& \mathbf{E}\left\{\overline{\left\langle\mathfrak{B}_{H, \boldsymbol{\xi}}, \boldsymbol{f}\right\rangle}\left\langle\boldsymbol{B}_{H, \boldsymbol{\xi}}, \boldsymbol{g}\right\rangle\right\}=\mathbf{E}\left\{\overline{\left\langle\epsilon_{H}(-\boldsymbol{\Delta})_{-\overline{\boldsymbol{\xi}}}^{-\frac{2 H+d}{4}} \mathfrak{W}, \boldsymbol{f}\right\rangle}\left\langle\epsilon_{H}(-\dot{\boldsymbol{\Delta}})_{-\overline{\boldsymbol{\xi}}}^{-\frac{2 H+d}{4}} \mathfrak{W}, \boldsymbol{g}\right\rangle\right\} \\
& =\left|\epsilon_{H}\right|^{2} \mathbf{E}\left\{\overline{\left\langle\mathfrak{W},(-\grave{\boldsymbol{\Delta}})_{-\boldsymbol{\xi}}^{-\frac{2 H+d}{4}} \boldsymbol{f}\right\rangle}\left\langle\mathfrak{W},(-\grave{\boldsymbol{\Delta}})_{-\boldsymbol{\xi}}^{-\frac{2 H+d}{4}} \boldsymbol{g}\right\rangle\right\} \\
& =\left|\epsilon_{H}\right|^{2}\left\langle\left\langle(-\grave{\Delta})_{-\boldsymbol{\xi}^{4}}^{-\frac{2 H+d}{4}} \boldsymbol{f},(-\grave{\boldsymbol{\Delta}})_{-\boldsymbol{\xi}}^{-\frac{2 H+d}{4}} \boldsymbol{g}\right\rangle_{\mathfrak{W}}\right. \\
& =\left|\epsilon_{H}\right|^{2}\left\langle(-\grave{\Delta})_{-\boldsymbol{\xi}}^{-\frac{2 H+d}{4}} \boldsymbol{f},(-\grave{\Delta})_{-\boldsymbol{\xi}}^{-\frac{2 H+d}{4}} \boldsymbol{g}\right\rangle \\
& =\frac{\left|\epsilon_{H}\right|^{2}}{(2 \pi)^{d}} \int_{\mathbb{R}^{d}} \mathrm{~d} \boldsymbol{\omega}\left[\mathbf{R}^{\frac{2 H+d}{4}} \hat{\boldsymbol{f}}\right]^{\mathrm{H}}(\boldsymbol{\omega}) \boldsymbol{\Phi}_{-2 \operatorname{Re}}^{-H-\frac{d}{2}} \boldsymbol{\xi}(\boldsymbol{\omega})\left[\mathbf{R}^{\frac{2 H+d}{4}} \hat{\boldsymbol{g}}\right](\boldsymbol{\omega}) ;
\end{aligned}
$$

(Parseval)

where we have used the following fact/definition regarding the correlation form of white Gaussian noise:

$$
\left\langle\langle\boldsymbol{f}, \boldsymbol{g}\rangle_{\mathfrak{W}}=\langle\boldsymbol{f}, \boldsymbol{g}\rangle .\right.
$$

\section{WAVELET ANALYSIS OF VECTOR FBM}

As a consequence of the whitening relation dictated by (1), vector fBm fields can (in loose terms) be regarded as combinations of (non-stationary) fractional integrals of white noise, with fractional integration appearing in the inversion of $(-\boldsymbol{\Delta})_{\boldsymbol{\xi}}^{\gamma}$.

For the purpose of analyzing these fields, it is desirable to undo the integration process, in order to produce a stationarized random field. This can be achieved by the application of a wavelet transform to the field, as wavelets-owing to their vanishing moments-behave like smoothed differentiators. Another important property of such an analysis follows from the self-similarity of both the wavelets and the random fields under consideration, which results in a simple relation between the probability distributions of wavelet coefficients at different scales. Consequently, wavelet analysis offers an advantageous way of verifying the introduced models and estimating their parameters in practice. ${ }^{2,3,6,7,11-14}$

Stationarizing the models in the manner we have just described can be seen as an attempt to construct a multi-resolution approximation of the operator $(-\boldsymbol{\Delta})_{\xi}^{\gamma}$ by using wavelets that have the operator embedded in them. Since in this process we are dealing with vector quantities and operators, the said wavelets are in general represented as matrices (or equivalently as groups of vector-valued functions). 


\subsection{Scalar Wavelet Analysis of Vector fBm}

In a biorthogonal wavelet system the basis elements can be represented as $\psi_{n, \boldsymbol{k}} \in \mathrm{L}^{2}$ with dual elements $\tilde{\psi}_{n, \boldsymbol{k}} \in \mathrm{L}^{2}$ (where $n$ denotes the resolution and $\boldsymbol{k}$ indexes a lattice in $\mathbb{R}^{d}$ ), so that the $i$-th component of $\boldsymbol{B}_{H, \boldsymbol{\xi}}$ finds the representation

$$
\left[\boldsymbol{B}_{H, \boldsymbol{\xi}}\right]_{i}=\sum_{n, \boldsymbol{k}}\left\langle\left[\boldsymbol{B}_{H, \boldsymbol{\xi}}\right]_{i}, \tilde{\psi}_{n, \boldsymbol{k}}\right\rangle \psi_{n, \boldsymbol{k}}=\sum_{n, \boldsymbol{k}}\left\langle\boldsymbol{\mathfrak { B }}_{H, \boldsymbol{\xi}}, \hat{\boldsymbol{e}}_{i} \tilde{\psi}_{n, \boldsymbol{k}}\right\rangle \psi_{n, \boldsymbol{k}}
$$

In standard constructions, wavelets in a given sub-band at a given resolution $n$ are lattice shifts of one another:

$$
\psi_{n, \boldsymbol{k}}(\boldsymbol{x})=\psi_{n, \mathbf{0}}(\boldsymbol{x}-\boldsymbol{k})
$$

Consider the discrete random process $w_{n, i}$ defined by

$$
w_{n, i}[\boldsymbol{k}]:=\left\langle\boldsymbol{B}_{H, \boldsymbol{\xi}}, \hat{\boldsymbol{e}}_{i} \tilde{\psi}_{\boldsymbol{k}}\right\rangle
$$

Then

$$
w_{n, i}[\boldsymbol{k}]=\left\langle\epsilon_{H}(-\dot{\boldsymbol{\Delta}})_{-\overline{\boldsymbol{\xi}}}^{-\frac{2 H+d}{4}} \mathfrak{W}, \hat{\boldsymbol{e}}_{i} \tilde{\psi}_{n, \boldsymbol{k}}\right\rangle=\left\langle\epsilon_{H} \mathfrak{W},(-\grave{\boldsymbol{\Delta}})_{-\boldsymbol{\xi}}^{-\frac{2 H+d}{4}} \hat{\boldsymbol{e}}_{i} \tilde{\psi}_{n, \boldsymbol{k}}\right\rangle
$$

Assuming that $\tilde{\psi}_{n, k}$ has sufficiently many vanishing moments (Fourier-domain zeros at $\boldsymbol{\omega}=0$ ) so that $\mathbf{R}^{\frac{2 H+d}{4}} \hat{\boldsymbol{e}}_{i} \tilde{\psi}_{n, \boldsymbol{k}}=\hat{\boldsymbol{e}}_{i} \tilde{\psi}_{n, \boldsymbol{k}}$, we have

$$
(-\grave{\boldsymbol{\Delta}})_{-\boldsymbol{\xi}}^{-\frac{2 H+d}{4}} \hat{\boldsymbol{e}}_{i} \tilde{\psi}_{n, \boldsymbol{k}}=(2 \pi)^{-d} \int_{\mathbb{R}^{d}} \mathrm{~d} \boldsymbol{\omega} \mathrm{e}^{\mathrm{j} \boldsymbol{x}^{\top} \boldsymbol{\omega}}\left[\boldsymbol{\Phi}_{-\boldsymbol{\xi}}^{-\frac{2 H+d}{4}}(\boldsymbol{\omega})\right]_{i}^{\top} \hat{\tilde{\psi}}_{n, \boldsymbol{k}}(\boldsymbol{\omega}) \in \mathrm{L}^{2}
$$

It therefore becomes clear that the above inverse operator is shift-invariant over this particular subspace of functions (in contrast to the general case of functions with non-vanishing moments for which it is not, due to the space-dependent operation of $\mathbf{R}^{\frac{2 H+d}{4}}$ ). But then, since $\mathfrak{W}$ is stationary, the last argument and (4) together prove that in this case the discrete random process $w_{n, i}$ is stationary.

In other words, a wavelet analysis of vector fractional Brownian motion with wavelets whose moments of degrees up to $\lfloor H\rfloor$ vanish yields stationary coefficients at each resolution.

\subsection{Extension to Vector Wavelets}

Instead of using ordinary scalar wavelets to analyze vector $\mathrm{fBm}$, it is possible to define vector wavelets that provide a multi-resolution approximation of the fractional Laplacian operator $(-\boldsymbol{\Delta})_{\xi}^{\gamma}$ (in comparison, scalar wavelets approximate differentiators of some order). This purpose is achieved by constructing a multi-resolution hierarchy of vector splines and defining wavelets via the application of a fractional Laplacian to interpolating spline basis functions.

\subsubsection{Vector Splines}

In a manner similar to that in which polyharmonic splines and wavelets are defined, ${ }^{14}$ we construct a multiresolution analysis of $\left(\mathrm{L}^{d}\right)^{d}$ by first finding a localized version of the Green's function of $(-\boldsymbol{\Delta})_{\xi}^{\gamma}$. Towards this end, we first recall that the said Green's function satisfies

$$
(-\Delta)_{\xi}^{\gamma} \boldsymbol{\Gamma}=\delta \mathbf{I}
$$

which yields the equation

$$
\boldsymbol{\Phi}_{\boldsymbol{\xi}}^{\gamma}(\boldsymbol{\omega}) \hat{\boldsymbol{\Gamma}}(\boldsymbol{\omega})=\mathbf{I} \text {, almost everywhere. }
$$

Given that the matrix inverse of $\boldsymbol{\Phi}_{\xi}^{\gamma}(\boldsymbol{\omega})$ for $\boldsymbol{\omega} \neq \mathbf{0}$ is simply $\boldsymbol{\Phi}_{-\boldsymbol{\xi}}^{-\gamma}(\boldsymbol{\omega})$, we can conclude that the desired Green's function has the Fourier transform

$$
\boldsymbol{\Phi}_{-\boldsymbol{\xi}}^{-\gamma}(\boldsymbol{\omega}) \text { almost everywhere. }
$$


Much in the same way as in scalar wavelet theory, a localized and interpolating version of the Green's function can be obtained via dividing its Fourier expression by its periodization. A Fourier-domain definition of the Lagrange basis function $\mathbf{L}_{\xi}^{\gamma}$ is therefore

$$
\hat{\mathbf{L}}_{\boldsymbol{\xi}}^{\gamma}(\boldsymbol{\omega}):=\boldsymbol{\Phi}_{-\boldsymbol{\xi}}^{-\gamma}(\boldsymbol{\omega})\left[\sum_{\boldsymbol{k} \in \mathbb{Z}^{d}} \boldsymbol{\Phi}_{-\boldsymbol{\xi}}^{-\gamma}(\boldsymbol{\omega}-2 \pi \boldsymbol{k})\right]^{-1},
$$

which ensures that

$$
\mathbf{L}_{\xi}^{\gamma}(\boldsymbol{k})=\delta_{\boldsymbol{k}} \mathbf{I} \text { for } \boldsymbol{k} \in \mathbb{Z}^{d}
$$

(with $\delta_{k}$ denoting Kronecker's delta function).

With basis elements (multi-integer shifts of $\mathbf{L}_{\xi}^{\gamma}$ ) given as matrix-valued functions in the vector setting, linear expansions take vectors as coefficients. The approximation space $\mathcal{V}_{0}$ is therefore defined thus:

$$
\mathcal{V}_{0}=\left\{\sum_{\boldsymbol{k} \in \mathbb{Z}^{d}} \mathbf{L}_{\boldsymbol{\xi}}^{\gamma}(\bullet-\boldsymbol{k}) \boldsymbol{c}[\boldsymbol{k}] \mid \boldsymbol{c} \in\left(\ell^{2}\right)^{d}\right\}
$$

One can verify that $\mathbf{L}_{\xi}^{\gamma}$ is indeed refinable, with the Fourier transform of the refinement filter given by*

$$
\left[\hat{\mathbf{L}}_{\boldsymbol{\xi}}^{\gamma}(\boldsymbol{\omega})\right]^{-1} \hat{\mathbf{L}}_{\boldsymbol{\xi}}^{\gamma}(2 \boldsymbol{\omega})=2^{-2 \gamma}\left[\sum_{\boldsymbol{k} \in \mathbb{Z}^{d}} \boldsymbol{\Phi}_{-\boldsymbol{\xi}}^{-\gamma}(\bullet-2 \pi \boldsymbol{k})\right]\left[\sum_{\boldsymbol{k} \in \mathbb{Z}^{d}} \boldsymbol{\Phi}_{-\boldsymbol{\xi}}^{-\gamma}(2 \bullet-2 \pi \boldsymbol{k})\right]^{-1},
$$

which is a $2 \pi \mathbb{Z}^{d}$-periodic function over $\mathbb{R}^{d}$. Nested multi-resolution spaces are then defined by the relation

$$
\mathcal{V}_{n}:=\left\{\boldsymbol{f}\left(2^{-n} \bullet\right) \mid \boldsymbol{f}(\bullet) \in \mathcal{V}_{0}\right\} .
$$

\subsubsection{Vector Wavelets}

Our intention in this subsection is to introduce wavelets that can be used to analyze a vector field $f(\bullet)$ in such a way that analysis coefficients together can be seen as a multi-resolution representation of the fractional Laplacian of the field in question. In other words, a given wavelet coefficient $\boldsymbol{w}_{n}[\boldsymbol{k}]$ (with $n$ encoding the resolution and $k$ the position) is to represent the quantity

$$
\int_{\mathbb{R}^{d}} \mathrm{~d} \boldsymbol{x} \mathbf{K}\left(2^{n} \boldsymbol{x}-\boldsymbol{k}\right)(-\boldsymbol{\Delta})_{\boldsymbol{\xi}}^{\gamma} \boldsymbol{f}(\boldsymbol{x})
$$

where $\mathbf{K}$ is a localized matrix-valued function. The above integral can then be interpreted as a local measure of $(-\boldsymbol{\Delta})_{\xi}^{\gamma} \boldsymbol{f}(\boldsymbol{x})$, the locality or resolution of which depends on $n$. One typically imposes the additional requirements on the wavelets that they be orthogonal to lower resolution approximation spaces and span their orthogonal complements in spaces of higher resolutions.

The two-scale relation in this case takes the form

$$
\mathbf{L}_{\xi}^{\gamma}\left(2^{-1} \bullet\right)=\sum_{k \in \mathbb{Z}^{d}} \mathbf{L}_{\xi}^{\gamma}(\bullet-k) h[k],
$$

which is to say that the refinement filter $\boldsymbol{h}$ corresponds to a sequence of vectors. The Fourier transform $\boldsymbol{h}$ of a vector filter $\boldsymbol{h}$ is given by the relation

$$
\stackrel{\circ}{h}(\boldsymbol{\omega}):=\sum_{\boldsymbol{k} \in \mathbb{Z}^{d}} \mathrm{e}^{\mathrm{j} \boldsymbol{k}^{\top} \boldsymbol{\omega}} \boldsymbol{h}[\boldsymbol{k}] .
$$

A $2 \pi \mathbb{Z}^{d}$-periodic vector-valued function over $\mathbb{R}^{d}$ can be taken to be the Fourier transform of a vector filter. As such, to show that $\mathbf{L}_{\xi}^{\gamma}$ is refinable, we need to show that the refinement filter obtained by dividing the Fourier transform of $\mathbf{L}_{\boldsymbol{\xi}}^{\gamma}\left(2^{-1} \bullet\right)$ by that of $\mathbf{L}_{\xi}^{\gamma}(\bullet)$ is $2 \pi \mathbb{Z}^{d}$-periodic. 
It turns out that the requirements sketched above can be satisfied by defining $2^{d}-1$ mother wavelets (corresponding to the usual $2^{d}-1$ Cartesian cosets) as follows.

$$
\mathbf{W}_{\boldsymbol{\xi}, m}^{\gamma}(\bullet):=(-\boldsymbol{\Delta}) \frac{\gamma}{\overline{\boldsymbol{\xi}}} \mathbf{L}_{\boldsymbol{\xi}+\overline{\boldsymbol{\xi}}}^{2 \gamma}\left(\bullet-\boldsymbol{\varsigma}_{m}\right),
$$

with $\varsigma_{m}, 1 \leq m \leq 2^{d}-1$, denoting the $m$-th coset identifier (i.e. the vector corresponding to the binary representation of $m$ ). A Fourier-domain characterization of these wavelets is given by the relation

$$
\begin{aligned}
\hat{\mathbf{W}}_{\boldsymbol{\xi}, m}^{\gamma}(\boldsymbol{\omega}) & :=\boldsymbol{\Phi}_{\boldsymbol{\xi}}^{\gamma}(\boldsymbol{\omega}) \hat{\mathbf{L}}_{\boldsymbol{\xi}+\overline{\boldsymbol{\xi}}}^{2 \gamma}(\boldsymbol{\omega}) \mathrm{e}^{-\mathrm{j} \boldsymbol{\omega}^{\top} \boldsymbol{s}_{m}} \\
& =\boldsymbol{\Phi}_{-\boldsymbol{\xi}}^{-\gamma}(\boldsymbol{\omega})\left[\sum_{\boldsymbol{k} \in \mathbb{Z}^{d}} \boldsymbol{\Phi}_{-(\boldsymbol{\xi}+\overline{\boldsymbol{\xi}})}^{-2 \gamma}(\boldsymbol{\omega}-2 \pi \boldsymbol{k})\right]^{-1} \mathrm{e}^{-\mathrm{j} \boldsymbol{\omega}^{\top} \boldsymbol{s}_{m}} .
\end{aligned}
$$

\subsubsection{Vector Wavelet Analysis of Vector fBm}

Let us now assume a $\gamma \geq \frac{H}{2}+\frac{d}{4}$ and consider the vector wavelet coefficients of a vector fBm field $\mathfrak{B}_{H, \boldsymbol{\xi}}$ :

$$
\begin{aligned}
\boldsymbol{w}_{0, m}[\boldsymbol{k}] & =\int_{\mathbb{R}^{d}} \mathrm{~d} \boldsymbol{x}\left[\mathbf{W}_{\overline{\boldsymbol{\xi}}}^{\gamma}(\boldsymbol{x})\right]^{\mathrm{H}} \boldsymbol{B}_{H, \boldsymbol{\xi}}(\boldsymbol{x}) \\
& =\epsilon_{H} \int_{\mathbb{R}^{d}} \mathrm{~d} \boldsymbol{x}\left[(-\boldsymbol{\Delta})_{\mathbf{0}}^{\gamma-\frac{H}{2}-\frac{d}{4}} \mathbf{L}_{\boldsymbol{\xi}+\boldsymbol{\xi}^{\prime}}^{2 \gamma}(\boldsymbol{x})\right] \mathfrak{W}(\boldsymbol{x})
\end{aligned}
$$

which, similar to the previous analysis with scalar wavelets, shows that these wavelet analysis coefficients constitute a stationary vector-valued random field (defined over $\mathbb{Z}^{d}$ ) corresponding to filtered white noise.

\section{CONCLUSION}

We defined a broad family of fractional Brownian random vector fields by introducing a pseudo-differential operator that extends the fractional vector Laplacian, and positing it as the whitening operator of the said random fields. A wavelet analysis of the introduced random fields using standard wavelets was then studied, with emphasis put on the stationarizing effect of a wavelet transform on vector $\mathrm{fBm}$.

Next, we turned our attention to the construction of multi-resolution approximation spaces and wavelets that are directly linked to the extended fractional Laplacian operator, in the sense that an analysis based on the said wavelets provides in effect a multi-resolution representation of the fractional Laplacian of the vector field under investigation. This property was used to demonstrate the stationarizing effect of these novel vector wavelets on fractional Brownian vector fields.

These newly introduced wavelets, and the random fields to which they are inherently related, have many interesting properties which we have not examined in this brief overview. These properties, and their interplay will be the subject of our future investigations. Moreover, in the present study, we have not addressed the practical implementation of the proposed wavelet analysis on a digital computer. Nevertheless, we are aware of an interesting connection between these wavelets and an important special function (namely the Epstein zeta function) for which fast evaluation algorithms have been proposed in the literature.

\section{REFERENCES}

1. B. B. Mandelbrot, Gaussian Self-Affinity and Fractals: Globality, The Earth, $1 / f$ Noise, and $R / S$, vol. $\mathrm{H}$ of Selecta (Old or New), Springer, New York, 2002.

2. S. Mallat, "A theory for multiresolution signal decomposition: The wavelet representation," IEEE Trans. Pattern Anal. Mach. Intell. 11, pp. 674-693, July 1989.

3. P. Flandrin, "Wavelet analysis and synthesis of fractional Brownian motion," IEEE Trans. Inf. Theory 38, pp. 910-916, March 1992.

4. G. W. Wornell, "Wavelet-based representations for the $1 / f$ family of fractal processes," Proc. IEEE 81, pp. 1428-1450, October 1993.

5. B. Ninness, "Estimation of $1 / f$ noise," IEEE Trans. Inf. Theory 44(1), pp. 32-46, 1998. 
6. D. Veitch and P. Abry, "A wavelet-based joint estimator of the parameters of long-range dependence," IEEE Trans. Inf. Theory 45, pp. 878-897, April 1999.

7. Y. Meyer, F. Sellan, and M. Taqqu, "Wavelets, generalized white noise and fractional integration: The synthesis of fractional Brownian motion," J. Fourier Anal. Appl. 5(5), pp. 465-494, 1999.

8. B. B. Mandelbrot and J. W. Van Ness, "Fractional Brownian motions, fractional noises and applications," SIAM Review 10(4), pp. 422-437, 1968.

9. I. M. Gel'fand and N. Vilenkin, Applications of Harmonic Analysis, vol. IV of Generalized Functions, Academic Press, 1964. translated by Amiel Feinstein.

10. J.-P. Kahane, Some Random Series of Functions, Cambridge University Press, 2nd ed., 1985.

11. M. Farge, "Wavelet transforms and their applications to turbulence," Annu. Rev. Fluid Mech. 24, pp. 395-457, 1992.

12. E. Masry, "The wavelet transform of stochastic processes with stationary increments and its application to fractional Brownian motion," IEEE Trans. Inf. Theory 39, pp. 260-264, January 1993.

13. T. Blu and M. Unser, "Self-similarity: Part II-Optimal estimation of fractal processes," IEEE Trans. Signal Process. 55, pp. 1364-1378, April 2007.

14. P. D. Tafti, D. Van De Ville, and M. Unser, "Invariances, Laplacian-like wavelet bases, and the whitening of fractal processes," IEEE Trans. Image Process. 18, pp. 698-702, April 2009. 